\title{
MODEL RIGID CR SUBMANIFOLDS OF CR DIMENSION 1
}

\author{
Al Boggess, Laura Ann Glenn and Alexander Nagel
}

The following three classes of models of rigid submanifolds of higher type with CR dimension one are discussed: 1) A tube-like model that only depends on the real part of the holomorphic tangent coordinate; 2) a radial model that depends on the modulus of the holomorphic tangent coordinate and 3) a free model. The first and third models have a Lie group structure which is analyzed. A characterization of the hull of holomorphy of the first two models is presented along with a partial result on the hull of holomorphy of the third.

\section{Introduction.}

The analysis of function theory on domains or submanifolds of $\mathbb{C}^{n}$ often first requires the understanding of function theory on simpler model cases. A classic example of this is the work of Folland and Stein [FS] which shows how the Siegel upper half plane and its boundary can be used as a model for general strictly pseudoconvex domains and strictly pseudoconvex hypersurfaces. More recently, boundaries of Siegel domains which are defined by quadratic polynomials can be used as models for the determination of the local hull of holomorphy and the description of reproducing kernels on a type 2 submanifold of $\mathbb{C}^{n}$ (see [BN1], [BN2]). The success of the Siegel domains in modeling more general cases stems from the fact that the leading terms of the Taylor expansion of the defining functions for a type two submanifold are precisely the defining functions for the boundary of a Siegel domain.

In this paper, we discuss models that are appropriate for submanifolds of higher type. The analysis of the most general submanifold of higher type is complicated. Here, we focus on the simpler class of rigid submanifolds of higher type where the dimension of the holomorphic tangent space at each point is one. For this class, we discuss three models: A tube-like model that only depends on the real part of the holomorphic tangent coordinate; a radial model that depends on the modulus of the holomorphic tangent coordinate; and a free model which is the most general of our models. As we show, the tube-like and free models have a Lie group structure that are generalizations of the Lie group structure for the boundary of a Siegel domain. 
An important part of the theory of CR submanifolds is the phenomenon of CR extendability. For example, Baouendi, Rothschild, and Treves [BRT] have shown that CR functions on CR submanifolds of finite type always extend to a wedge. We show be example that the notion of wedge extendability does not behave well under restriction. If $U \subset V \subset M$ are open subsets of a CR submanifold, it may happen that every wedge of extendability for $U$ may be disjoint from any given wedge of extendability for $V$. Our example suggests that rather than linear wedges, it is more natural to study regions which are invariant under certain non-isotropic dilations. We do this for the model examples, and show how the models can approximate a general rigid CR submanifold.

For the tube-like model, we show that its polynomial hull is the same as its convex hull. This is a special case of some recent results of Boivin and Dwilewicz (see $[\mathrm{BD}]$ ) which state that the hull of holomorphy of a tube-like CR manifold is its convex hull (i.e. the analogue of Bochner's Tube Theorem for open sets, see theorem 2.5.10 in $[\mathbf{H}]$ ). In one direction, this result generalizes results in $[\mathrm{BN} 1]$ and $[\mathrm{BN2}]$ that show the hull of the boundary of the Siegel domain is its convex hull. Less information is available for the hulls of the radial and free models. Perhaps the most interesting result in this paper indicates how much we do not know about the hull of the free case. We prove an estimate for analytic discs with boundary in the free model that implies that the set of images of analytic discs with boundaries in the free model is strictly smaller than the convex hull of the free model. This result raises interesting unanswered questions concerning the exact nature of the hull of this simple model.

The paper is organized as follows. Section 2 reviews the basic definitions of rigid submanifolds and type. The third section reviews the definition of $\mathrm{CR}$ function and wedge extendability, and presents an example which shows the limitations of this notion under restriction. Section 4 contains a description of our three models and we show how a general CR manifold with $\mathrm{CR}$ dimension one can be modeled by the free model. Section 5 discusses the Lie group structures on the ultra-rigid and free models. Section 6 discusses the process of approximation of general rigid CR manifolds by complex linear images of the model manifolds. The polynomial hulls of the tube-like model, the radial model, and the free models are discussed in Sections 7, 8 and 9, respectively.

\section{Rigid CR submanifolds of CR dimension 1 and of finite type.}

Our object of study is the class of rigid CR submanifolds $M \subset \mathbb{C}^{n}$ of finite type and of $\mathrm{CR}$ dimension 1 . We describe this class in terms of local coordi- 
nates in the following way. Suppose $n=d+1$ and let $\Phi=\left(\phi_{1}, \ldots, \phi_{d}\right): \mathbb{C} \rightarrow$ $\mathbb{R}^{d}$ be a smooth mapping. Write points in $\mathbb{C} \times \mathbb{C}^{d}$ as $(z, w)=\left(z, w_{1}, \ldots, w_{d}\right)$, and we write $z=x+\imath y, w_{j}=u_{j}+\imath v_{j}$. A CR submanifold $M \subset \mathbb{C}^{d+1}$ is rigid and has $C R$ dimension 1 if locally there are coordinates so that $M$ has the form

$$
\begin{aligned}
M & =\left\{(z, w) \in \mathbb{C} \times \mathbb{C}^{d} \mid \operatorname{Re}(w)=\Phi(z)\right\} \\
& =\left\{(z, w) \in \mathbb{C} \times \mathbb{C}^{d} \mid u_{j}=\phi_{j}(z)=\phi_{j}(x+\imath y) \quad 1 \leq j \leq d\right\} .
\end{aligned}
$$

Note that $M$ is rigid because we can choose defining functions for $M$ that are independent of the coordinates $\left\{v_{1}, \ldots, v_{d}\right\}$. For a more intrinsic definition of rigid submanifold, see Baouendi, Rothschild, and Treves [BRT].

In terms of these coordinates, the complexified holomorphic tangent bundle of $M$ is spanned (over the ring of smooth, complex valued functions on $M)$ by the vector fields $L$ and $\bar{L}$ where

$$
L=\frac{\partial}{\partial z}+2 \sum_{k=1}^{d} \frac{\partial \phi_{k}}{\partial z}(z) \frac{\partial}{\partial w_{k}} \quad \text { and } \quad \bar{L}=\frac{\partial}{\partial \bar{z}}+2 \sum_{k=1}^{d} \frac{\partial \phi_{k}}{\partial \bar{z}}(z) \frac{\partial}{\partial \bar{w}_{k}} .
$$

The totally real tangent bundle to $M$ is spanned (over the ring of smooth, complex valued functions on $M$ ) by the vector fields $\left\{\frac{\partial}{\partial v_{1}}, \ldots, \frac{\partial}{\partial v_{d}}\right\}$. The complexified tangent bundle $T^{\mathbb{C}}(M)$ is therefore spanned by $L, \bar{L}$, and $\left\{\frac{\partial}{\partial v_{1}}, \ldots, \frac{\partial}{\partial v_{d}}\right\}$.

Note that

$$
[L, \bar{L}]=2 \sum_{k=1}^{d} \frac{\partial^{2} \phi_{k}}{\partial z \partial \bar{z}}(z)\left[\frac{\partial}{\partial \bar{w}_{k}}-\frac{\partial}{\partial w_{k}}\right]=2 \imath \sum_{k=1}^{d} \frac{\partial^{2} \phi_{k}}{\partial z \partial \bar{z}}(z) \frac{\partial}{\partial v_{k}} .
$$

Write $L=L^{+1}$ and $\bar{L}=L^{-1}$. Then for any $N$-tuple $\left\{i_{1}, \cdots, i_{N}\right\}$ of plus and minus ones, we have the following formula for the iterated commutator of length $N+2$

$$
\left[L^{i_{1}},\left[L^{i_{2}},\left[\cdots\left[L^{i_{N}},[L, \bar{L}]\right] \cdots\right] \cdots\right]\right]=2 \imath \sum_{k=1}^{d} \frac{\partial^{\alpha+\beta}}{\partial z^{\alpha} \partial \bar{z}^{\beta}}\left(\frac{\partial^{2} \phi_{k}(z)}{\partial z \partial \bar{z}}\right) \frac{\partial}{\partial v_{k}} .
$$

Here $\alpha$ is the number of plus ones and $\beta$ is the number of minus ones in the sequence $\left\{i_{1}, \cdots, i_{N}\right\}$. Thus the Lie algebra generated by $L$ and $\bar{L}$ is spanned by the vector fields $\left\{L, \bar{L},[L, \bar{L}], \ldots,[L, \bar{L}]^{(\alpha, \beta)}, \ldots\right\}$ where

$$
[L, \bar{L}]^{(\alpha, \beta)}=\sum_{k=1}^{d} \frac{\partial^{\alpha+\beta}}{\partial z^{\alpha} \partial \bar{z}^{\beta}}\left(\frac{\partial^{2} \phi_{k}(z)}{\partial z \partial \bar{z}}\right) \frac{\partial}{\partial v_{k}},
$$

and $[L, \bar{L}]=[L, \bar{L}]^{(0,0)}$. 
For $p \in M$, let $\mathcal{L}_{p}^{1}(M)$ be the vector subspace of $T_{p}^{\mathbb{C}}(M)$ spanned by $L$ and $\bar{L}$, and for $j \geq 2$, let $\mathcal{L}_{p}^{j}(M)$ be the vector subspace of $T_{p}^{\mathbb{C}}(M)$ spanned by $L$ and $\bar{L}$ and all Lie brackets $[L, \bar{L}]^{(\alpha, \beta)}$ with $\alpha+\beta \leq j-2$. Clearly, $\operatorname{dim}_{\mathbb{C}} \mathcal{L}_{p}^{j}(M) \leq \operatorname{dim}_{\mathbb{C}} \mathcal{L}_{p}^{j+1}(M)$. Following Bloom and Graham [BG], we say that $\left(m_{1}, \ldots, m_{d}\right)$ with $m_{i} \leq m_{j} \leq+\infty$ for $i<j$ are the Hörmander numbers for $M$ at $p$ if

$$
m_{i}=\inf \left\{j \mid \operatorname{dim}_{\mathbb{C}} \mathcal{L}_{p}^{j}(M) \geq 2+i\right\} .
$$

$M$ is said to be of finite type $m$ at $p$ if $\mathcal{L}_{p}^{m}(M)=T_{p}^{\mathbb{C}}(M)$, but $\mathcal{L}_{p}^{m-1}(M) \neq$ $T_{p}^{\mathbb{C}}(M)$. In this case $m=m_{d}<+\infty$.

For any integer $N$ we can write

$$
\phi_{j}(z)=\sum_{\alpha, \beta \leq N} a_{\alpha, \beta}^{j}\left(z_{0}\right)\left(z-z_{0}\right)^{\alpha}\left(\bar{z}-\bar{z}_{0}\right)^{\beta}+E_{N}^{j}\left(z, z_{0}\right)
$$

where, since the functions $\phi_{j}$ are real valued, $\overline{a_{\alpha, \beta}^{j}\left(z_{0}\right)}=a_{\beta, \alpha}^{j}\left(z_{0}\right)$, and $\left|E_{N}^{j}\left(z, z_{0}\right)\right| \leq C_{N}\left|z-z_{0}\right|^{N+1}$. Moreover, this error estimate is uniform for $p=\left(z_{0}, w_{0}\right)$ in a compact subset of $M$.

For each $p=\left(z_{0}, w_{0}\right)$, we can make a biholomorphic change of variables (depending smoothly on $p$ ) such that the Taylor polynomial of degree $N$ of each $\phi_{j}$ has no "pure terms"; i.e. that

$$
a_{\alpha, \beta}^{j}\left(z_{0}\right)=0 \quad \text { if either } \alpha=0 \text { or } \beta=0 .
$$

(See Boggess [B, 7.2, Theorem 1].) In particular, we may assume that at the point $p=\left(z_{0}, w_{0}\right)$, the space $\mathcal{L}_{p}^{1}(M)$ is spanned by $\left\{\frac{\partial}{\partial z}, \frac{\partial}{\partial \bar{z}}\right\}$. Let $Y_{j}\left(z_{0}\right)$ denote the subspace of $\mathbb{C}^{d}$ generated by the set of vectors

$$
\left\{\frac{\partial^{\alpha+\beta}}{\partial z^{\alpha} \partial \bar{z}^{\beta}}\left(\frac{\partial^{2} \Phi}{\partial z \partial \bar{z}}\right)\left(z_{0}\right)\right\}, \quad 0 \leq \alpha+\beta \leq j-2 .
$$

Under an obvious identification, the space $\mathcal{L}_{p}^{j}(M)$ is spanned by $\left\{\frac{\partial}{\partial z}, \frac{\partial}{\partial \bar{z}}\right\}$ and the space $Y_{j}\left(z_{0}\right)$. Thus $M$ is of finite type $m$ at a point $\left(z_{0}, w_{0}\right)$ if and only if the collection of vectors $\left\{\frac{\partial^{\alpha+\beta}}{\partial z^{\alpha} \partial \bar{z}^{\beta}}\left(\frac{\partial^{2} \Phi\left(z_{0}\right)}{\partial z \partial \bar{z}}\right), 0 \leq \alpha+\beta \leq m-2\right\}$, span $\mathbb{C}^{d}$. In terms of the Taylor coefficients of the functions $\phi_{k}, M$ is of finite type $m$ at a point $\left(z_{0}, w_{0}\right)$ if and only if the vectors $\left(a_{\alpha \beta}^{1}, \ldots, a_{\alpha \beta}^{d}\right), \quad 2 \leq \alpha+\beta \leq m$, span $\mathbb{C}^{d}$. Note that for a rigid manifold of $\mathrm{CR}$ dimension one with coordinates chosen so that the functions $\phi_{k}$ have no pure terms, the Hörmander numbers at the point $p=\left(z_{0}, w_{0}\right)$ are just the degrees of the leading order terms of the Taylor expansions of the $\phi_{k}^{\prime} s$ (assuming that they are arranged in increasing order). 
The type and dimensions of the spaces $\mathcal{L}_{\left(z_{0}, w_{0}\right)}^{j}(M)$ may vary from point to point due to changing linear relations between the vector fields $[L, \bar{L}]^{(\alpha, \beta)}$. We are interested in constructing examples where this does not happen. It is convenient for us to consider a slightly different set of vectors. Set

$$
Z_{j}\left(z_{0}\right)=\left\{\frac{\partial^{\alpha+\beta}}{\partial z^{\alpha} \partial \bar{z}^{\beta}}\left(\frac{\partial^{2} \Phi\left(z_{0}\right)}{\partial z \partial \bar{z}}\right) \in \mathbb{C}^{d}, \quad 0 \leq \alpha \leq j-1,0 \leq \beta \leq j-1\right\} .
$$

It is clear that $M$ is of finite type at $z_{0}$ if and only if the span of $Z_{j}\left(z_{0}\right)=\mathbb{C}^{d}$ for some $j$. In fact, if $M$ is of type $2 m$ at $z_{0}$, then $Z_{m}\left(z_{0}\right)=\mathbb{C}^{d}$, and if $Z_{m}\left(z_{0}\right)=\mathbb{C}^{d}$, then $M$ is of type $2 m$ at $z_{0}$.

Definition 2.1. $\quad M \subset \mathbb{C}^{d+1}$ is an $m$-free rigid generic $C R$ submanifold of $C R$ dimension one if the vector fields $\left\{[L, \bar{L}]^{\alpha, \beta}, 0 \leq \alpha, \beta \leq m-1\right\}$ are linearly independent (i.e. no relations exist between these vector fields), and they span $\mathbb{C}^{d}$.

In Section 4 , we shall describe a model manifold $F_{m}$ which is m-free and of type $2 m$.

\section{CR Extendability, Wedge Extendability, and an Example.}

A function or distribution on a generic CR submanifold $M \subset \mathbb{C}^{n}$ is a $C R$ function if it is annihilated by all tangential vector fields of type $(0,1)$. In the special case of manifolds of CR dimension 1 as discussed in the last section, this simply means that the function or distribution $f$ satisfies $\bar{L}(f)=$ 0 . In many cases, CR distributions are the boundary values on $M$ (in an appropriate sense) of holomorphic functions defined in open sets $\Omega \subset \mathbb{C}^{n}$ which contain $M$ in its boundary.

In particular, it is a special case of a theorem of Baouendi, Rothschild and Treves [BRT] that if $M$ is a generic rigid CR submanifold of $\mathbb{C}^{n}$, every CR function defined in a neighborhood of a point $p \in M$ of finite type extends holomorphically to a wedge. In the context of Section 2, this means the following. Let $\Gamma$ be an open convex cone in $\mathbb{R}^{d}$. For any $\epsilon>0$, let $\Gamma^{\epsilon}$ be the elements of $\Gamma$ of length less than $\epsilon$. Let $V \subset M$ be an open set. Then a wedge $\mathcal{W}_{\Gamma^{\epsilon}}(V)$ with edge $V \subset M$ is the set of the form

$$
\mathcal{W}_{\Gamma^{\epsilon}}(V)=\left\{(z, w) \in \mathbb{C}^{d+1} \mid \operatorname{Re}(w)-\Phi(z)=u \in \Gamma^{\epsilon}, \quad(z, w-u) \in V\right\} .
$$

A precise statement of a special case of the theorem of Baouendi, Rothschild and Treves is the following:

Theorem [BRT]. Suppose that $0 \in M$ is a point of finite type, and that $U$ is a neighborhood of 0 in $M$. Then there is a cone $\Gamma \subset \mathbb{R}^{d}$, an open 
neighborhood $V \subset U$ of 0 in $M$, and an $\epsilon>0$ such that every CR function defined on $U$ extends to a holomorphic function on $W_{\Gamma^{\epsilon}}(V)$.

This result gives an important result on CR extendability. However one difficulty with the notion of wedge-extendability is that is does not behave well as one shrinks the open set $U$. Unlike the case of hypersurfaces, shrinking the open set $U$ does not necessarily lead to shrinking of the wedge of extendability. Instead, the wedge of extendability may 'rotate' as the open set shrinks. We give an example of this phenomenon for a rigid CR submanifolds of CR dimension 1.

Example 3.1. Let

$$
M=\left\{\left(z, w_{1}, w_{2}\right) \in \mathbb{C}^{3} \mid \operatorname{Re}\left(w_{1}\right)=[\operatorname{Re}(z)]^{2}, \quad \operatorname{Re}\left(w_{2}\right)=[\operatorname{Re}(z)]^{4}\right\} .
$$

Given an open set $U$ containing the origin, choose any cone $\Gamma$, parameter $\epsilon>$ 0 and open subset $V \subset U$ on which we have wedge extendability according to [BRT]. Then there exists an open set $\tilde{U} \subset V$ containing the origin so that every cone $\tilde{\Gamma}$ on which we have wedge extendability for $\tilde{U}$ is disjoint from the cone $\Gamma$.

To see why this is so, note that the region of extendability of CR functions from an open subset $V \subset M$ is always contained in the linear convex hull of the set $V$. Thus the above example follows from the following simple observation:

Lemma 3.2. Let $M_{\delta}=\left\{\left(x+\imath y_{1}, x^{2}+\imath y_{2}, x^{4}+\imath y_{3}\right) \in \mathbb{C}^{3}|| x \mid<\delta\right\}$. Let $\hat{M}_{\delta}$ denote the linear convex hull of $M_{\delta}$. Then if $\left(z_{1}, z_{2}, z_{3}\right) \in \hat{M}_{\delta}$, it follows that

$$
\left(\operatorname{Re}\left[z_{2}\right]\right)^{2} \leq \operatorname{Re}\left[z_{3}\right] \leq \delta^{2} \operatorname{Re}\left[z_{2}\right]
$$

Proof. Let $z^{j}=\left(x_{j}+\imath y_{1, j}, x_{j}^{2}+\imath y_{2, j}, x_{j}^{4}+\imath y_{3, j}\right), 1 \leq j \leq N$ be $N$ points of $\hat{M}_{\delta}$. Let $\lambda_{j} \geq 0,1 \leq j \leq N$ satisfy $\sum_{j=1}^{N} \lambda_{j}=1$ and let $\left(z_{1}, z_{2}, z_{3}\right)=$ $\sum_{j=1}^{N} \lambda_{j}\left(z_{1}^{j}, z_{2}^{j}, z_{3}^{j}\right)$. Then

$$
\begin{aligned}
\operatorname{Re}\left[z_{2}\right] & =\sum_{j=1}^{N} \lambda_{j} x_{j}^{2} \leq\left(\sum_{j=1}^{N} \lambda_{j}\right)^{1 / 2}\left(\sum_{j=1}^{N} \lambda_{j} x_{j}^{4}\right)^{1 / 2}=\left(\sum_{j=1}^{N} \lambda_{j} x_{j}^{4}\right)^{1 / 2} \\
& =\left(\operatorname{Re}\left[z_{3}\right]\right)^{1 / 2} \leq \delta\left(\sum_{j=1}^{N} \lambda_{j} x_{j}^{2}\right)^{1 / 2}=\delta\left(\operatorname{Re}\left[z_{2}\right]\right)^{1 / 2}
\end{aligned}
$$

which proves the lemma. 
To demonstrate the assertion in example (3.1), let $U$ be any open set containing the origin on $M$. Then the cone for wedge extendability for $U$ must be given by

$$
\Gamma=\left\{\left(x_{2}, x_{3}\right) \mid 0<\alpha \leq \frac{x_{3}}{x_{2}} \leq \beta<+\infty\right\}
$$

for some $\alpha$ and $\beta$. If we then choose $\delta$ so that $\delta^{2}<\alpha$, and let

$$
\tilde{U}=\left\{\left(x+\imath y_{1}, x^{2}+\imath y_{2}, x^{4}+\imath y_{3}\right) \in V|| x \mid<\delta\right\},
$$

then Lemma 3.2 shows that the cone for wedge extendability for $\tilde{U}$ must be disjoint from $\Gamma$.

This example suggests that the concept of wedge extendability, which involves isotropic cones, may not give the optimal results on CR extendability. Rather, the example indicates that CR extendability takes place in non-isotropic cones which reflect different homogeneities in directions which require different numbers of commutators. Since in general these directions can vary from point to point, we study certain model examples where this cannot happen.

\section{Three Classes of Model Submanifolds.}

In this section, we introduce three classes of model CR submanifolds: Tube models, $T_{m}$, radial models, $R_{m}$, and free models, $F_{m}$.

\subsection{Tube-like models $T_{m}$.}

The simplest model for a CR manifold of finite type with CR dimension one is the tube model, $T_{m}$, which is defined by

$$
T_{m}=\left\{\left(z_{1}, z_{2}, \ldots, z_{m}\right) \in \mathbb{C}^{m} \mid \operatorname{Re}\left(z_{j}\right)=\left[\operatorname{Re}\left(z_{1}\right)\right]^{j}, \quad 2 \leq j \leq m\right\} .
$$

For this simple model, we have re-labeled the coordinates from Section 2 (with $z_{1}$ playing the role of $z$ and $z_{j}$ playing the role of $w_{j-1}$ ). $T_{m}$ is a real submanifold of $\mathbb{C}^{m}$ of real dimension $m+1$ and real codimension $m-1$. If we write the coordinates as $z_{j}=x_{j}+\imath y_{j}$, then there is a unique (up to scalar multiple) global holomorphic tangential vector field on $T_{m}$ given by

$$
L=\frac{\partial}{\partial z_{1}}+\sum_{j=2}^{m} j\left(\operatorname{Re} z_{1}\right)^{j-1} \frac{\partial}{\partial z_{j}} .
$$

In particular, $T_{m}$ is a $\mathrm{CR}$ submanifold of $\mathbb{C}^{m}$ of $\mathrm{CR}$ dimension 1. An easy calculation shows that the Hörmander numbers of $T_{m}$ at the origin are $(2, \ldots, m)$ and the type of $T_{m}$ at the origin is $m$. In Section 5 , we shall describe a Lie Group structure on $T_{m}$ for which $L$ is left invariant. Therefore, all points of $T_{m}$ have the same Hörmander numbers and type. 
4.2. The radial model, $R_{m}$.

The radial model, $R_{m}$, is defined by

$$
R_{m}=\left\{\left.\left(z, w_{1}, w_{2}, \ldots, w_{m}\right) \in \mathbb{C}^{m}\left|\operatorname{Re}\left(w_{j}\right)=\right| z\right|^{2 j}, \quad 1 \leq j \leq m\right\} .
$$

The generator for the holomorphic tangent bundle of $R_{m}$ is

$$
L=\frac{\partial}{\partial z}+2 \sum_{k=1}^{m} k z^{k-1} \bar{z}^{k} \frac{\partial}{\partial w_{k}} .
$$

This submanifold of $\mathbb{C} \times \mathbb{C}^{m}$ has real dimension $m+2$ and real codimension $m$. The Hörmander numbers for $R_{m}$ at the origin are $(2,4, \ldots 2 m)$. However, the Hörmander numbers for $R_{m}$ change as the point changes (for example, if $m=2$, the Hörmander numbers change from $(2,4)$ at the origin to $(2,3)$ away from the origin, as the reader can easily verify by computing $[L,[L, \bar{L}]])$. This means that there is no Lie group structure $(z \cdot w)$ on $R_{m}$ which is holomorphic in $w$.

\subsection{The free model, $F_{m}$.}

In discussing this last class of examples, it is convenient to identify the complex space, $\mathbb{C}^{m^{2}}$, with the space, $M_{m}^{\mathbb{C}}$, of $m \times m$ matrices with complex entries. There are two natural ways of splitting $M_{m}^{\mathbb{C}}$ into "real" and "imaginary" parts. The first way is to decompose a matrix $W \in M_{m}^{\mathbb{C}}$ as

$$
W=\operatorname{Re}(W)+\imath \operatorname{Im}(W)
$$

where the $i, j^{t h}$ entry of $\operatorname{Re}(W)$ and $\operatorname{Im}(W)$ is the real and imaginary parts, respectively, of the $i, j^{t h}$ entry of $W$. The second way involves a decomposition into Hermitian parts. For $W \in M_{m}^{\mathbb{C}}$, let

$$
\begin{aligned}
& \Re(W)=\frac{1}{2}\left(W+W^{*}\right), \\
& \Im(W)=\frac{1}{2 \imath}\left(W-W^{*}\right) .
\end{aligned}
$$

Clearly, $\Re(W), \Im(W)$ belong to the space, $H_{m}$, of Hermitian $m \times m$ matrices and

$$
W=\Re(W)+\imath \Im(W) .
$$

Note that for scalar $(m=1), \Re(W)=\operatorname{Re}(W)$ and $\Im(W)=\operatorname{Im}(W)$. These two ways of decomposing $W$ are related by a nonsingular, complex linear change of coordinates. In fact, let $w_{j k}$ and $u_{j k}, 1 \leq j, k \leq m$, be two sets of coordinates for $M_{m}^{\mathbb{C}}$ and define the map $Q: M_{m}^{\mathbb{C}} \mapsto M_{m}^{\mathbb{C}}$ by $u=Q(w)$, where

$$
\begin{array}{ll}
u_{j j}=w_{j j} & 1 \leq j \leq m, \\
u_{j k}=\frac{w_{j k}+w_{k j}}{2} & 1 \leq j<k \leq m, \\
u_{j k}=\frac{w_{j k}-w_{k j}}{2 \imath} & 1 \leq k<j \leq m .
\end{array}
$$


It is an easy exercise to show that

$$
\begin{aligned}
& Q \circ \Re=\operatorname{Re} \circ Q \\
& Q \circ \Im=\operatorname{Im} \circ Q .
\end{aligned}
$$

Thus, in the case of a rigid manifold $M$ given by

$$
M=\left\{(z, w) \in \mathbb{C} \times \mathbb{C}^{m^{2}} \mid \operatorname{Re}(w)=\Phi(z)\right\}
$$

we shall often rewrite the defining equation for $M$ as

$$
M=\left\{(z, w) \in \mathbb{C} \times M_{m}^{\mathbb{C}} \mid \Re(w)=\tilde{\Phi}(z)\right\}
$$

where, now, $\tilde{\Phi}$ is a smooth map from $\mathbb{C}$ to $H_{m}$ and is related to $\Phi$ by the equation $\Phi=Q \circ \tilde{\Phi}$.

We now turn to the definition of the model. Define $\Phi_{0}: \mathbb{C} \rightarrow H_{m}$ by

$$
\Phi_{0}(z)=\left[\begin{array}{cccc}
z \bar{z} & z \bar{z}^{2} & \cdots & z \bar{z}^{m} \\
z^{2} \bar{z} & z^{2} \bar{z}^{2} & \cdots & z^{2} \bar{z}^{m} \\
\vdots & \vdots & \ddots & \vdots \\
z^{m} \bar{z} & z^{m} \bar{z}^{2} & \cdots & z^{m} \bar{z}^{m}
\end{array}\right]
$$

The $m$-free model, $F_{m}$ is defined by

$$
F_{m}=\left\{(z, W) \in \mathbb{C} \times M_{m}^{\mathbb{C}} \mid \Re(W)=\Phi_{0}(z)\right\} .
$$

Note that we are using the second way of presenting a free manifold that is discussed at the beginning of this section. Using the change of variables $u=Q(w)$, we can re-write the defining equations of $F_{m}$ as $(4.3 .2)^{\prime}$

$$
F_{m}=\left\{\left(z,\left[u_{j k}\right]\right) \in \mathbb{C} \times \mathbb{C}^{m^{2}} \mid \operatorname{Re}\left(u_{j k}\right)=\left\{\begin{array}{ll}
\operatorname{Re}\left(z^{j} \bar{z}^{k}\right), & \text { for } 1 \leq j<k \leq m \\
|z|^{2 j}, & \text { for } 1 \leq j=k \leq m \\
\operatorname{Im}\left(z^{j} \bar{z}^{k}\right), & \text { for } 1 \leq k<j \leq m
\end{array}\right\} .\right.
$$

We shall usually work with the defining equations given in the original presentation of $F_{m}$ in order to avoid having to deal with the separate cases of $j<k$ and $j>k$. Written out in components, the $m^{2}$ defining functions for $F_{m}$ are

$$
\rho_{j k}(z, w)=\frac{1}{2}\left(w_{j k}+\bar{w}_{k j}\right)-z^{j} \bar{z}^{k}=0 .
$$

The space of $(1,0)$-vectors is spanned by the vector field

$$
L=\frac{\partial}{\partial z}+\sum_{j, k=1}^{m} 2 j z^{j-1} \bar{z}^{k} \frac{\partial}{\partial w_{j k}} .
$$


Thus

$$
[L, \bar{L}]=\sum_{j, k=1}^{m} 2 j k z^{j-1} \bar{z}^{k-1} T_{j k}
$$

where

$$
T_{j k}=\frac{\partial}{\partial \bar{w}_{k j}}-\frac{\partial}{\partial w_{j k}} .
$$

Note that $T_{j k}\left(\rho_{l m}\right) \equiv 0$ for all $j, k, l, m$, so the vectors $\left\{T_{j k}\right\}$ belong to the complexified tangent space to $F_{m}$ at every point. These vectors are also linearly independent, and so the vectors $\left\{L, \bar{L}, \ldots, T_{j k}, \ldots\right\}, 1 \leq j, k \leq m$, span the complexified tangent space to $F_{m}$ at every point.

Let us write $L^{+1}=L$ and $L^{-1}=\bar{L}$. For an $N$ - tuple $\left\{i_{1}, \cdots, i_{N}\right\}$ of plus and minus ones, let $\alpha$ and $\beta$ denote the number of plus ones and minus ones, respectively. If $\alpha \leq m-1$ and $\beta \leq m-1$ then the iterated commutator is given by

$$
\begin{aligned}
& {\left[L^{i_{1}},\left[L^{i_{2}},\left[\cdots\left[L^{i_{N}}[L, \bar{L}]\right] \cdots\right] \cdots\right]\right]} \\
& =2 \sum_{j=\alpha+1}^{m} \sum_{k=\beta+1}^{m} \frac{j !}{(j-\alpha-1) !} \frac{k !}{(k-\beta-1) !} z^{j-\alpha-1} \bar{z}^{k-\beta-1} T_{j k} \\
& \equiv[L, \bar{L}]^{(\alpha, \beta)}
\end{aligned}
$$

Note, the commutator does not depend on the order of the $L^{i_{j}}$ 's. The Lie algebra generated by $L$ and $\bar{L}$ is spanned by the vector fields $\{L, \bar{L}, \ldots$, $\left.[L, \bar{L}]^{\alpha, \beta}, \ldots\right\}$ with $0 \leq \alpha \leq m-1,0 \leq \beta \leq m-1$.

Proposition 4.3.1. $\quad F_{m}$ is an $m$-free rigid submanifold of type $2 \mathrm{~m}$.

Proof. It suffices to show that the set of $m^{2}$-vector fields $[L, \bar{L}]^{\alpha, \beta} 0 \leq \alpha, \beta \leq$ $m-1$ are linearly independent. Suppose there were complex constants $c_{\alpha, \beta}$ and a point $z_{0}$ so that

$$
\sum_{\alpha, \beta=0}^{m-1} c_{\alpha, \beta}[L, \bar{L}]^{\alpha, \beta}\left(z_{0}\right)=0
$$

It follows that

$$
\sum_{j=1}^{m} \sum_{k=1}^{m}\left[\sum_{\alpha=0}^{j-1} \sum_{\beta=0}^{k-1} \frac{j !}{(j-\alpha-1) !} \frac{k !}{(k-\beta-1) !} z_{0}^{j-1-\alpha} \bar{z}_{0}^{k-1-\beta} c_{\alpha, \beta}\right] T_{j k}=0 .
$$


Since the collection of $\left\{T_{j k}\right\}$ is linearly independent, we must have

$$
\sum_{\alpha=0}^{j-1} \sum_{\beta=0}^{k-1} \frac{j !}{(j-\alpha-1) !} \frac{k !}{(k-\beta-1) !} z_{0}^{j-1-\alpha} \bar{z}_{0}^{k-1-\beta} c_{\alpha, \beta}=0
$$

for $1 \leq j \leq m$ and $1 \leq k \leq m$. This is a homogeneous system of $m^{2}$ equations in the $m^{2}$ unknowns $\left\{c_{\alpha, \beta}\right\}$. In fact, it is really a "triangular" system with non-zero entries along the diagonal. Thus setting $j=k=1$, we see that $c_{00}=0$. For any $\alpha_{0}, \beta_{0}$ suppose by induction that $c_{\alpha \beta}=0$ if either $\alpha=\alpha_{0}$ and $\beta<\beta_{0}$ or $\alpha<\alpha_{0}$ and $\beta \leq \beta_{0}$. Then the equation with $j=\alpha_{0}+1$ and $k=\beta_{0}+1$ shows that $c_{\alpha_{0} \beta_{0}}=0$. This shows that the vector fields $[L, \bar{L}]^{\alpha, \beta}$ are linearly independent, as desired.

\section{The nilpotent Lie group structures of $T_{m}$ and $F_{m}$.}

When $m=1$

$$
T_{1}=F_{1}=\left\{\left(z_{1}, z_{2}\right) \in \mathbb{C}^{2} \mid \operatorname{Re}\left(z_{2}\right)=\left[\operatorname{Re}\left(z_{1}\right)\right]^{2}\right\}
$$

It is easy to see that this set is biholomorphic to the set

$$
H^{1}=\left\{\left.\left(w_{1}, w_{2}\right)\left|\operatorname{Re}\left(w_{2}\right)=\right| w_{1}\right|^{2}\right\}
$$

under the change of variables $w_{1}=z_{1}$ and $w_{2}=2 z_{2}-z_{1}^{2}$. It is well known that $H^{1}$ carries the structure of a nilpotent Lie group, the Heisenberg group. The object of this section is to show that the classes of models $T_{m}$ and $F_{m}$ also carry nilpotent Lie group structures which are compatible with their complex structure, and which generalize the case $m=1$.

\subsection{The group structure for $T_{m}$.}

In this section, we define a product $z \cdot w$ for $z, w \in \mathbb{C}^{m} \times \mathbb{C}^{m}$ which is holomorphic in $w$ in such a way that $T_{m}$ becomes a closed Lie subgroup. We motivate the definition of the product as follows. We write $z=\left(z_{1}, \ldots, z_{m}\right)$ and $w=\left(w_{1}, \ldots, w_{m}\right)$. As with the product on the Heisenberg group, we want the first component of the product $z \cdot w$ to be $z_{1}+w_{1}$. Therefore, we set $z \cdot w=\left(z_{1}+w_{1}, c_{2}, c_{3}, \ldots, c_{m}\right)$ where the $c_{j}$ must be chosen so that $z \cdot w$ belongs to $T_{m}$ provided $z, w \in T_{m}$. This means that we should require $\Re\left(c_{k}\right)=\left(\Re\left(z_{1}\right)+\Re\left(w_{1}\right)\right)^{k} \quad 2 \leq k \leq m$. Expanding this equation, we obtain

$$
\Re\left(c_{k}\right)=\left(\Re z_{1}\right)^{k}+\left(\sum_{j=1}^{k-1}\left(\begin{array}{l}
k \\
j
\end{array}\right)\left(\Re z_{1}\right)^{k-j}\left(\Re w_{1}\right)^{j}\right)+\left(\Re w_{1}\right)^{k} .
$$


If $z$ and $w$ belong to $T_{m}$, then $\Re z_{j}=\left(\Re z_{1}\right)^{j}$ and $\Re w_{j}=\left(\Re w_{1}\right)^{j}$, and we obtain

$$
\Re\left(c_{k}\right)=\Re\left(z_{k}\right)+\left(\sum_{j=1}^{k-1}\left(\begin{array}{l}
k \\
j
\end{array}\right) \Re\left(z_{k-j}\right) \Re\left(w_{j}\right)\right)+\Re\left(w_{k}\right) .
$$

Since we wish $z \cdot w$ to be holomorphic in $w$, we set

$$
z \cdot w=\left(z_{1}+w_{1}, c_{2}, c_{3}, \ldots, c_{m}\right)
$$

where

$$
c_{k}=z_{k}+w_{k}+\sum_{j=1}^{k-1}\left(\begin{array}{l}
k \\
j
\end{array}\right) \Re\left(z_{k-j}\right) w_{j} .
$$

\section{Lemma 5.1.}

(1) Under the product $\cdot \mathbb{C}^{m}$ is a Lie Group.

(2) For each fixed $z \in \mathbb{C}^{m}$, the map $w \mapsto T_{z}(w)=z \cdot w$ is a nonsingular complex affine (and hence biholomorphic) map from $\mathbb{C}^{m}$ to itself.

(3) The set $T_{m}$ is a closed Lie subgroup and the vector field $L$ given in equation (4.1.2) is a left invariant vector field. The Lie subalgebra of the group $T_{m}$ is the Lie algebra generated by the vector fields $L$ and $\bar{L}$.

Proof. To show (1), we first establish the associative property. We denote the $k^{t h}$ coordinate of $z \cdot w$ by $(z \cdot w)_{k}$. Applying the definition of the product, we have

$$
\begin{aligned}
(z \cdot(w \cdot u))_{k}=\left[z_{k}+w_{k}+u_{k}\right] & +\sum_{j=1}^{k-1}\left(\begin{array}{c}
k \\
j
\end{array}\right) \Re\left(z_{k-j}\right) w_{j} \\
& +\sum_{j=1}^{k-1}\left(\begin{array}{c}
k \\
j
\end{array}\right) \Re\left(z_{k-j}\right) u_{j} \\
& +\sum_{l=1}^{k-1}\left(\begin{array}{c}
k \\
l
\end{array}\right) \Re\left(w_{k-l}\right) u_{l} \\
& +\sum_{j=2}^{k-1} \sum_{l=1}^{j-1}\left(\begin{array}{c}
k \\
j
\end{array}\right)\left(\begin{array}{l}
j \\
l
\end{array}\right) \Re\left(z_{k-j}\right) \Re\left(w_{j-l}\right) u_{l} .
\end{aligned}
$$


On the other hand, we have

$$
\begin{aligned}
((z \cdot w) \cdot u)_{k}=\left[z_{k}+w_{k}+u_{k}\right] & +\sum_{j=1}^{k-1}\left(\begin{array}{c}
k \\
j
\end{array}\right) \Re\left(z_{k-j}\right) w_{j} \\
& +\sum_{j=1}^{k-1}\left(\begin{array}{c}
k \\
j
\end{array}\right) \Re\left(z_{k-j}\right) u_{j} \\
& +\sum_{l=1}^{k-1}\left(\begin{array}{c}
k \\
l
\end{array}\right) \Re\left(w_{k-l}\right) u_{l} \\
& +\sum_{l=1}^{k-2} \sum_{j=1}^{k-l-1}\left(\begin{array}{c}
k \\
l
\end{array}\right)\left(\begin{array}{c}
k-l \\
j
\end{array}\right) \Re\left(z_{k-l-j}\right) \Re\left(w_{j}\right) u_{l} .
\end{aligned}
$$

It suffices to show the two double sums on the right sides of the above expansions are equal. To equate these double sums, make the substitution $j^{\prime}=j+l$ in the index of summation in the double sum for $((z \cdot w) \cdot u)_{k}$. Then switch the order of summation. This completes the proof that the product - is associative.

Note that the origin is the identity element for this multiplication. An easy computation shows that the inverse of $\left(z_{1}, \ldots, z_{m}\right)$ is the point $\left(w_{1}, \ldots, w_{m}\right)$ where

$$
w_{k}=-z_{k}-\sum_{j=1}^{k-1}\left(\begin{array}{l}
k \\
j
\end{array}\right) \Re(z)_{k-j} w_{j}
$$

This completes the proof of (1). The proof of (2) is easy and left to the reader.

For (3), we showed that $T_{m}$ is closed under the product - as part of the motivation and derivation of the formula for this product. Therefore to show $T_{m}$ is a closed Lie subgroup, it suffices to show that the inverse of a point $z \in T_{m}$ also belongs to $T_{m}$. This is easily seen by showing that the point $w=z^{-1}$ given above satisfies the defining equations, $\Re w_{k}=\left(\Re w_{1}\right)^{k}$, for $M$ provided $z$ satisfies these equations.

Now, we show that the vector field (4.1.2), $L=\frac{\partial}{\partial z_{1}}+\sum_{j=2}^{m} j\left(\Re z_{1}\right)^{j-1} \frac{\partial}{\partial z_{j}}$ is left invariant under the action of the group. This means that we must show that the differential of the map $T_{z}: T_{m} \rightarrow T_{m}$ sends the vector $L$ at the point $w$ to the vector $L$ at the point $z \cdot w$; or in other words, we must show $L_{w}\left\{f \circ T_{z}\right\}=L_{z \cdot w}\{f\}$. We first show this to be true for $w=0$. At the origin, the vector $L$ is just $\frac{\partial}{\partial w_{1}}$. We have

$$
L_{0}\left\{f \circ T_{z}\right\}=\left.\frac{\partial}{\partial w_{1}}\{f(z \cdot w)\}\right|_{w=0}=\frac{\partial f(z)}{\partial z_{1}}+\sum_{j=2}^{m} j\left[\Re\left(z_{1}\right)\right]^{j-1} \frac{\partial f(z)}{\partial z_{j}}=L(f)(z) .
$$


The case for nonzero $w$, now follows from the associativity of the group action (i.e. $T_{z} \circ T_{w}=T_{z \cdot w}$ ). Indeed, using what we just proved for $w=0$, we have

$$
L_{w}\left\{f \circ T_{z}\right\}=L_{0}\left\{f \circ T_{z} \circ T_{w}\right\}=L_{0}\left\{f \circ T_{z \cdot w}\right\}=L_{z \cdot w}\{f\}
$$

as desired.

\subsection{The group structure for $F_{m}$.}

Now, we define a product $(z, A) \cdot(w, B)$ on the space $\mathbb{C} \times M_{m}^{\mathbb{C}}$ which is holomorphic in $(w, B)$ in such a way that $F_{m}$ becomes a closed Lie subgroup. This product will be a generalization of the product on the Heisenberg group (the case $m=1$ ). We shall motivate the form of the product in a similar manner to the motivation given for the formula for the product on $T_{m}$. Let $(z, A),(w, B)$ be elements of $\mathbb{C} \times M_{m}^{\mathbb{C}}$ with

$$
A=\left[\begin{array}{ccc}
a_{11} & \cdots & a_{1 m} \\
\vdots & \ddots & \vdots \\
a_{m 1} & \cdots & a_{m m}
\end{array}\right] \text { and } B=\left[\begin{array}{ccc}
b_{11} & \cdots & b_{1 m} \\
\vdots & \ddots & \vdots \\
b_{m 1} & \cdots & b_{m m}
\end{array}\right]
$$

As with the Heisenberg group, we wish the first component of $(z, A) \cdot(w, B)$ to be $z+w$. So we set $(z, A) \cdot(w, B)=(z+w, C)$ where $C$ is the matrix

$$
C=\left[\begin{array}{ccc}
c_{11} & \cdots & c_{1 m} \\
\vdots & \ddots & \vdots \\
c_{m 1} & \cdots & c_{m m}
\end{array}\right]
$$

which must be chosen so that $(z+w, C)$ belongs to $F_{m}$ if $(z, A)$ and $(w, B)$ belong to $F_{m}$. In order for $(z+w, C)$ to belong to $F_{m}$, the $j, k^{t h}$ component of $\Re(C)$ must be set equal to $(z+w)^{j} \overline{(z+w)}^{k}$. Expanding this equation, we obtain

$$
\begin{aligned}
\Re(C)_{j k} & =\left(z^{j}+\sum_{\ell=1}^{j}\left(\begin{array}{l}
j \\
\ell
\end{array}\right) z^{j-\ell} w^{\ell}\right)\left(\bar{z}^{k}+\sum_{r=1}^{k}\left(\begin{array}{l}
k \\
r
\end{array}\right) \bar{z}^{k-r} \bar{w}^{r}\right) \\
& =z^{j} \bar{z}^{k}+z^{j} \sum_{r=1}^{k}\left(\begin{array}{l}
k \\
r
\end{array}\right) \bar{z}^{k-r} \bar{w}^{r}+\bar{z}^{k} \sum_{\ell=1}^{j}\left(\begin{array}{l}
j \\
\ell
\end{array}\right) z^{j-\ell} w^{\ell} \\
& +\sum_{\ell=1}^{j} \sum_{r=1}^{k}\left(\begin{array}{l}
j \\
\ell
\end{array}\right)\left(\begin{array}{l}
k \\
r
\end{array}\right) z^{j-\ell} \bar{z}^{k-r} w^{\ell} \bar{w}^{r} .
\end{aligned}
$$

If $(z, A)$ and $(w, B)$ belong to $F_{m}$, we have $\Re(A)_{j k}=z^{j} \bar{z}^{k}$ and $\Re(B)_{\ell_{r}}=$ $w^{\ell} \bar{w}^{r}$. We observe that the second term on the right is the conjugate of the 
third term with $j$ replaced by $k$. Moreover, the first and fourth terms are Hermitian. Thus for general $(z, A)$ and $(w, B)$ set

$$
(z, A) \cdot(w, B)=(z+w, C)
$$

with

$$
c_{j k}=a_{j k}+\sum_{\ell=1}^{j} \sum_{r=1}^{k}\left(\begin{array}{l}
j \\
\ell
\end{array}\right)\left(\begin{array}{l}
k \\
r
\end{array}\right) z^{j-\ell} \bar{z}^{k-r} b_{\ell r}+2\left[\sum_{\ell=1}^{j}\left(\begin{array}{l}
j \\
\ell
\end{array}\right) z^{j-\ell} w^{\ell}\right] \bar{z}^{k}
$$

It is now an easy exercise to show that if $(z, A)$ and $(w, B)$ belong to $F_{m}$ then $\Re(C)_{j k}=(z+w)^{j} \overline{(z+w)}^{k}$.

\section{Lemma 5.2.}

(1) Under the operation $\cdot$, the space $\mathbb{C} \times M_{m}^{\mathbb{C}}$ is a nilpotent Lie group.

(2) For each fixed $(z, A) \in \mathbb{C} \times M_{m}^{\mathbb{C}}$ the mapping

$$
(w, B) \rightarrow T_{(z, A)}(w, B)=(z, A) \cdot(w, B)
$$

is a nonsingular complex affine (and hence biholomorphic) mapping of $\mathbb{C} \times M_{m}^{\mathbb{C}}$ to itself.

(3) The set $F_{m}$ is a closed Lie subgroup. The Lie algebra of the Lie group $F_{m}$ is the same as the Lie algebra generated by the left invariant vector fields $L$ and $\bar{L}$ defined in Section 4.3 .

Proof. We first sketch the proof of (1). The associative property is established by an argument that is similar in spirit to the proof that the product on $T_{m}$ is associative, so we shall leave it as an exercise. As in the tube-case, the identity element is $(0,0)$. It is easy to show that the inverse of $(w, B)$ is the point $(z, A)$ where $z=-w$ and the $j, k^{t h}$ element of $A$ is given by

$$
\begin{aligned}
a_{j k}=-2(-1)^{k} \bar{w}^{k} \sum_{\ell=1}^{j}\left(\begin{array}{l}
j \\
\ell
\end{array}\right)(-1)^{j-\ell} w^{j} & \\
& -\sum_{\ell=1}^{j} \sum_{r=1}^{k}\left(\begin{array}{l}
j \\
\ell
\end{array}\right)\left(\begin{array}{l}
k \\
r
\end{array}\right)(-1)^{j-\ell+k-r} w^{j-\ell} \bar{w}^{k-r} b_{\ell r} .
\end{aligned}
$$

This completes the proof of (1). The proof of (2) is easy and left to the reader.

For (3), we motivated the formula for the product - so that $F_{m}$ is closed. We leave it as an exercise to show that if $(w, B)$ belongs to $F_{m}$, then its inverse, $(z, A)$ given above, also belongs to $F_{m}$. 
It remains to show that the vector field (4.3.3),

$$
L=\frac{\partial}{\partial z}+\sum_{j, k=1}^{m} 2 j z^{j-1} \bar{z}^{k} \frac{\partial}{\partial w_{j k}}
$$

is left invariant under the group action. This means that we must show that the differential of the map $T_{(z, A)}: F_{m} \rightarrow F_{m}$ sends the vector $L$ at the point $(w, B)$ to the vector $L$ at the point $(z, A) \cdot(w, B)$. Using the associativity of the group action, it suffices to show this for $(w, B)=(0,0)$. At the origin, the vector $L$ is just $\frac{\partial}{\partial w}$. We have

$$
\begin{aligned}
& \left.L_{0}\left\{T_{(z, A)} f(w, B)\right\}\right|_{(w, B)=(0,0)} \\
& =\left.\frac{\partial}{\partial w}\{f((z, A) \cdot(w, B))\}\right|_{(w, B)=(0,0)} \\
& =\frac{\partial f}{\partial z}(z, A)+\left.\sum_{j, k=1}^{m} \frac{\partial f(z, A)}{\partial w_{j k}} \cdot \frac{\partial}{\partial w}\left\{c_{j k}\right\}\right|_{(w, B)=(0,0)}
\end{aligned}
$$

where $c_{j k}$ is the $j, k$-entry of the matrix $C$ resulting from the product $(z, A)$. $(w, B)=(z+w, C)$. We therefore obtain

$$
\left.L_{0}\left\{T_{(z, A)} f(w, B)\right\}\right|_{(w, B)=(0,0)}=\frac{\partial f}{\partial z}(z, A)+\sum_{j, k=1}^{m} 2 j z^{j-1} \bar{z}^{k} \frac{\partial f(z, A)}{\partial w_{j k}} .
$$

In view of the formula (4.3.3) for $L$, this expression is just $L_{(z, A)}\{f\}$, as desired. This completes the proof of (3).

\section{Approximation by the models.}

In this section, we show how a general rigid CR submanifold with $\mathrm{CR}$ dimension one can be approximated by a complex linear image of the model $F_{m}$. Let $M$ be a smooth rigid $\mathrm{CR}$ submanifold with $\mathrm{CR}$ dimension 1 given by

$$
M=\left\{(z, w) \in \mathbb{C} \times \mathbb{C}^{d} \mid \operatorname{Re}(w)=\Phi(z)\right\}
$$

where $\Phi: \mathbb{C} \rightarrow \mathbb{R}^{d}$ is smooth. We suppose that $M$ is of finite type at most $2 m$ at the origin where $m \geq 1$ is an integer. We write $\phi_{j}(z)=$ $\sum_{\alpha, \beta \leq m} a_{\alpha, \beta}^{j} z^{\alpha} \bar{z}^{\beta}+E_{m}^{j}(z)$, where $\overline{a_{\alpha, \beta}^{j}}=a_{\beta, \alpha}^{j} \in \mathbb{C}$, and $E_{m}^{j}$ is an error term of order $m+1$. Put $E_{m}(z)=\left(E_{m}^{1}(z), \ldots, E_{m}^{d}(z)\right)$. As in Section 2, we assume $a_{\alpha, \beta}^{j}=0$ if either $\alpha=0$ or $\beta=0$. The finite type hypothesis means that the set of vectors $\left\{\left(a_{\alpha \beta}^{1}, a_{\alpha \beta}^{2}, \ldots, a_{\alpha \beta}^{d}\right) \in \mathbb{C}^{d}, 1 \leq \alpha \leq m, 1 \leq \beta \leq m\right\}$ span $\mathbb{C}^{d}$. 
Define the complex linear map $\Psi: M_{m}^{\mathbb{C}} \rightarrow \mathbb{C}^{d}$ by writing $\Psi=\left(\psi_{1}, \ldots, \psi_{d}\right)$, where

$$
\psi_{j}\left(\left[w_{\alpha \beta}\right]\right)=\sum_{\alpha=1}^{m} \sum_{\beta=1}^{m} a_{\alpha, \beta}^{j} w_{\alpha \beta} \quad 1 \leq j \leq d .
$$

The finite type hypothesis implies that the mapping $\Psi$ is surjective. We have

$$
\overline{\psi_{j}\left(\left[w_{\alpha \beta}\right]\right)}=\sum_{\alpha=1}^{m} \sum_{\beta=1}^{m} \overline{a_{\alpha, \beta}^{j}} \overline{w_{\alpha \beta}}=\sum_{\alpha=1}^{m} \sum_{\beta=1}^{m} a_{\alpha, \beta}^{j} \overline{w_{\beta \alpha}}
$$

and hence $\operatorname{Re}\left(\psi_{j}(W)\right)=\psi_{j}(\Re(W))$. Thus

$$
\Psi: H_{m} \rightarrow \mathbb{R}^{d} \subset \mathbb{C}^{d} .
$$

Set

$$
\mathcal{X}=\left\{[A] \in H_{m} \mid \psi([A])=0\right\} .
$$

Let $\tilde{M}$ be the image of the model submanifold $F_{m}$ under the complex linear mapping $(I \times \Psi)(z, W)=(z, \Psi(W))$. If $(z, W)=\left(z, \Phi_{0}(z)+\imath Y\right) \in F_{m}$ then for $1 \leq j \leq d$,

$$
\operatorname{Re}\left(\psi_{j}(W)\right)=\psi_{j}(\Re[W])=\psi_{j}\left(\Phi_{0}(z)\right)=\sum_{\alpha=1}^{m} \sum_{\beta=1}^{m} a_{\alpha, \beta}^{j} z^{\alpha} \bar{z}^{\beta} .
$$

Thus $\tilde{M}$ is also a rigid CR submanifold of $C^{d+1}$, and is given by

$$
\tilde{M}=\left\{(z, w) \in \mathbb{C}^{d+1} \mid \operatorname{Re}(w)=\Psi \circ \Phi_{0}(z)\right\}
$$

where $\Phi_{0}$ was defined in (4.3.1). Note that because

$$
\Phi(z)-\Psi \circ \Phi_{0}(z)=E_{m}(z)
$$

the manifold $\tilde{M}$ is tangent to the original manifold $M$ at the origin to order $2 m$. Define a map $j: M \rightarrow \tilde{M}$ by setting

$$
j(z, \Phi(z)+\imath V)=\left(z, \Psi \circ \Phi_{0}(z)+\imath V\right) .
$$

Next we show that we can also "free" the manifold $M$ by adding variables. Let $\left\{A_{d+1}, \ldots, A_{m^{2}}\right\}$ be a basis for $\mathcal{X}$, and let $a_{\alpha \beta}^{j}$ denote the $\alpha, \beta^{\text {th }}$ entry of $A_{j}$ for $d+1 \leq j \leq m^{2}$. Set

$$
\begin{aligned}
\hat{M}=\left\{\left(z, w_{1}, \ldots, w_{d}, w_{d+1}, \ldots, w_{m^{2}}\right) \in \mathbb{C}^{1+m^{2}} \mid\right. \\
\left.\operatorname{Re}\left(w_{j}\right)=\left\{\begin{array}{ll}
\phi_{j}(z) & \text { for } 1 \leq j \leq d \\
\sum_{\alpha, \beta=1}^{m} a_{\alpha \beta}^{j} z^{\alpha} \bar{z}^{\beta} & \text { for } d+1 \leq j \leq m^{2}
\end{array}\right\}\right\} .
\end{aligned}
$$


It is easy to show that $\hat{M}$ is an m-free generic CR submanifold of CR dimension 1. We let $\Pi: \mathbb{C} \times \mathbb{C}^{m^{2}} \rightarrow \mathbb{C} \times \mathbb{C}^{d}$ be the projection map onto the first $d+1$ coordinates (given by $\left(z, w_{1}, \ldots, w_{d}\right)$ ). Clearly, $\Pi$ maps $\hat{M}$ onto $M$. We let $\hat{\Psi}: H_{m} \mapsto \tilde{\mathbb{C}}^{m^{2}}$ be the map analogous to $\Psi$ whose $j^{\text {th }}$ coordinate is

$$
\hat{\psi}_{j}\left(\left[w_{\alpha \beta}\right]\right)=\sum_{\alpha=1}^{m} \sum_{\beta=1}^{m} a_{\alpha, \beta}^{j} w_{\alpha \beta} \quad 1 \leq j \leq m^{2} .
$$

Let $\tilde{\hat{M}}=(I \times \hat{\Psi})\left(F_{m}\right)$, and let $\hat{j}: \hat{M} \rightarrow \tilde{\hat{M}}$ be defined as in (6.9). We have proved the following.

Proposition 6.1. Given a rigid $\mathrm{CR}$ submanifold $M \subset \mathbb{C} \times \mathbb{C}^{d}$ of $\mathrm{CR}$ dimension 1 , with $0 \in M$ and of finite type at most $2 m$ at 0 , there is an $m$-free manifold $\hat{M} \subset \mathbb{C}^{1+m^{2}}$, a holomorphic projection map $\Pi: \mathbb{C} \times \mathbb{C}^{m^{2}} \rightarrow \mathbb{C} \times \mathbb{C}^{d}$ and complex linear maps $I \times \Psi: \mathbb{C} \times \mathbb{C}^{m^{2}} \rightarrow \mathbb{C} \times \mathbb{C}^{d}$ and $I \times \hat{\Psi}: \mathbb{C} \times \mathbb{C}^{m^{2}} \rightarrow$ $\mathbb{C} \times \mathbb{C}^{m^{2}}$ such that the following holds.

(1) $(I \times \Psi)\left\{F_{m}\right\}=\tilde{M}$ is a complex linear image of the model $F_{m}$ and it approximates $M$ to order $m$ at the origin.

(2) $(I \times \hat{\Psi})\left\{F_{m}\right\}=\hat{\tilde{M}}$ is a complex linear image of the model $F_{m}$ and it approximates $\tilde{M}$ to order $m$ at the origin.

(3) The following diagram commutes:

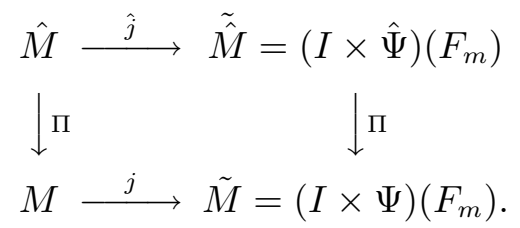

In principle, we can now use this approximation technique to transfer information about the model manifolds to information about a general rigid manifold. A typical example of this would be information about the local hull of holomorphy of $M$, or questions about local extendability of CR functions. This is because such questions are often studied by means of analytic discs, and these discs behave very well under the approximation scheme described above. We quickly review the notion of analytic discs.

Suppose $M$ is a submanifold of $\mathbb{C}^{n}$. Fix a number $0<\alpha<1$ ( $\alpha$ equal to $1 / 2$ will do). Let $D$ denote the open unit disc in $\mathbb{C}$ with coordinate $\zeta$. An analytic disc with boundary in $M$ is an analytic map $A: D \rightarrow \mathbb{C}^{n}$ such that $A$ is of class $C^{\alpha}$ (Hölder continuous with exponent $\alpha$ ) up to the boundary of $D$ and such that the image of the unit circle under $A$ belongs to $M$. By the 
maximum principle, the polynomial hull of any set $M$ is contained in the set of images of analytic discs with boundaries in $M$. By re-parameterizing an analytic disc, it suffices to consider the set of "centers" of analytic discs. Thus we define the disc set for $M$, denoted by Disc $(M)$, as

$$
\left\{z \in \mathbb{C}^{n} \mid z=A(\zeta=0) \text { for some analytic } \operatorname{disc} A \text { with boundary in } M\right\} .
$$

Consider the special case of a rigid manifold, $M$ defined by $M=\{(z, w) \in$ $\left.\mathbb{C} \times \mathbb{C}^{d} ; \operatorname{Re}(w)=\Phi(z)\right\}$. If an analytic disc $A=(f, g): D \rightarrow \mathbb{C} \times \mathbb{C}^{d}$ has boundary in $M$, then $f$ and $g$ must satisfy the equation

$$
\operatorname{Re}(g(\zeta))=\Phi(f(\zeta)) \quad \text { for }|\zeta|=1 .
$$

Conversely, given an analytic function $f: D \rightarrow \mathbb{C}$ which is of class $C^{\alpha}$ up to the unit circle, then the above equation uniquely determines the real part of an analytic function $g$ on $D$. Using the Hilbert transform, the imaginary part of $g$ is uniquely determined up to an additive constant $y \in \mathbb{R}^{m}$. Note that the real part of $g$ belongs to the class $C^{\alpha}$ and since the Hilbert transform is continuous in the $C^{\alpha}$ norm, $\operatorname{Im}(g)$ will also be of class $C^{\alpha}$ up to the unit circle. Therefore, an analytic function $f: D \rightarrow \mathbb{C}$ of class $C^{\alpha}$ up to the unit circle and a vector $y \in \mathbb{R}^{d}$, generate an analytic disc $A=(f, g)$ with boundary in $M$ such that $\operatorname{Im}(g(\zeta=0))=y$.

Now let $f$ be an analytic function on the unit disc $D$ which is of class $C^{\alpha}$ up to the unit circle, and let $y \in \mathbb{R}^{d}$. Let $\hat{y} \in \mathbb{R}^{m^{2}}$ be the vector whose first $d$ entries are given by $y$ and all other entries are 0 . Then $f$ and $y$ generate analytic discs $A=(f, g)$ and $\tilde{A}=(f, \tilde{g})$ for the manifolds $M$ and its approximation $\tilde{M}$. (Due to the high order of tangency, if $f$ is small, the two discs will in fact be very close to each other and we can estimate the difference between $g$ and $\tilde{g}$.) Similarly, $f$ and $\hat{y}$ generate analytic discs $\hat{A}=(f, \hat{g})$ and $\tilde{\hat{A}}=(f, \tilde{\hat{g}})$ for the manifolds $\hat{M}$ and $\tilde{\hat{M}}$. It is clear from the construction (or from uniqueness) that we have:

Proposition 6.2. $\Pi \circ \hat{A}=A$ and $\Pi \circ \tilde{\hat{A}}=\tilde{A}$.

Moreover the analytic function and the vector $\hat{y}$ (regarded as a Hermitian matrix) also determine an analytic disc in the model $F_{m}$. Thus if we can characterize Disc $\left(F_{m}\right)$, then $\operatorname{Disc}(\tilde{M})$ and $\operatorname{Disc}(\tilde{\hat{M}})$ are given as complex linear images of this set, and these in turn approximate Disc $(M)$ and Disc $(\hat{M})$ near the origin. This motivates the study of the disc set of the model $F_{m}$.

In the foregoing discussion, we have considered approximating a general rigid $\mathrm{CR}$ submanifold of finite type and $\mathrm{CR}$ dimension one. However, if the 
manifold has additional symmetry properties (such as $\Phi$ depending only on $\operatorname{Re}(z)$ or depending only on $|z|)$, then we can use the models $T_{m}$ or $R_{m}$ instead of $F_{m}$. As we shall see, understanding the disc sets of these models is considerably easier than the problem of understanding the disc set of $F_{m}$.

\section{The Polynomial Hull of $T_{m}$.}

Recall that the polynomial hull of a set $M \subset \mathbb{C}^{n}$ is the set

$$
\left\{z \in \mathbb{C}^{n} ;|p(z)| \leq \sup _{M}|p| \text { for all polynomials } p\right\} .
$$

The main theorem of this section is the following.

Theorem 7.1. The polynomial hull of $T_{m} \subset \mathbb{C}^{m}$ is the convex hull of $T_{m}$.

Theorem 7.1 will follow from the following theorem on the disc set for $T_{m}$, defined in (6.11).

\section{Theorem 7.2.}

(1) $\operatorname{Disc}\left(T_{m}\right)$ is contained in the convex hull of $T_{m}$.

(2) The convex hull of $T_{m}$ is contained in the closure of $\operatorname{Disc}\left(T_{m}\right)$.

(3) $\operatorname{Disc}\left(T_{m}\right)-T_{m}$ is an open set.

The above theorem and its proof imply that Disc $\left(T_{m}\right)-T_{m}$ is equal to the interior of the convex hull of $T_{m}$.

Proof of Theorem 7.2. We begin by describing the disc set of $T_{m}$. If $A=$ $(f, g)=\left(f, g_{2}, \ldots, g_{m}\right): D \rightarrow \mathbb{C}^{m}$ is an analytic disc with boundary in $T_{m}$, then we must have

$$
\operatorname{Re}\left[g_{j}(\zeta)\right]=[\operatorname{Re}[f(\zeta)]]^{j}, \quad|\zeta|=1,2 \leq j \leq m .
$$

The functions $g_{j}$ are uniquely determined up to an additive imaginary constant by the real part of $f$. Write $f=u+\imath v$, and write $\operatorname{Im}\left[g_{j}(0)\right]=y_{j}$. Keep in mind that we are at liberty to choose the analytic disc $f$ and the vector $y \in \mathbb{R}^{m}$. By the Poisson integral formula, $A(\zeta=0)$ is given by the point

$$
\begin{aligned}
&\left(\frac{1}{2 \pi} \int_{0}^{2 \pi} u\left(e^{\imath \theta}\right) d \theta+\imath y_{1}, \frac{1}{2 \pi} \int_{0}^{2 \pi} u\left(e^{\imath \theta}\right)^{2} d \theta+\imath y_{2}, \ldots\right. \\
&\left.\frac{1}{2 \pi} \int_{0}^{2 \pi} u\left(e^{\imath \theta}\right)^{m} d \theta+\imath y_{m}\right) .
\end{aligned}
$$

Set

$$
\begin{array}{r}
\mathcal{W}_{m}=\left\{\left(x_{1}, x_{2}, \ldots, x_{m}\right) \in \mathbb{R}^{m} \mid \text { there exists } u \in C^{\alpha}([0,2 \pi])\right. \text { with } \\
\left.x_{j}=\frac{1}{2 \pi} \int_{0}^{2 \pi}\left[u\left(e^{\imath \theta}\right)\right]^{j} d \theta, \quad 1 \leq j \leq m\right\} .
\end{array}
$$


Thus we have shown that

$$
\operatorname{Disc}\left(T_{m}\right)=\mathcal{W}_{m}+\imath \mathbb{R}^{m} .
$$

Denote the convex hull of the set $\left\{\left(t, t^{2}, \ldots t^{m}\right) \in \mathbb{R}^{m} \mid t \in \mathbb{R}\right\}$ by $\Gamma_{m}$. Since the defining functions for $T_{m}$ are independent of $y=\operatorname{Im}(z)$, to prove Theorem 7.2 we must show that:

(1) $\mathcal{W}_{m} \subset \Gamma_{m}$;

(2) $\Gamma_{m}$ is contained in the closure of $\mathcal{W}_{m}$;

(3) Disc $\left(T_{m}\right)-T_{m}$ is an open set.

The problem of characterizing the set $\mathcal{W}_{m}$ can be viewed from the point of view of measure theory by characterizing the set of "moment" sequences $x_{1}, \ldots, x_{m}$, where

$$
x_{j}=\int_{\mathbb{R}} t^{j} d \nu(t)
$$

for some Borel probability measure $\nu$ on $\mathbb{R}$. However, we shall give a geometric solution to the problem as originally stated.

Lemma 7.3. The closure of the interior of $\mathcal{W}_{m}$ is $\Gamma_{m}$.

Proof. First, we show that $\mathcal{W}_{m}$ is contained in $\Gamma_{m}$. Let $\left(\xi_{1}, \xi_{2}, \ldots, \xi_{m}\right)$ be a vector in $\mathbb{R}^{m}$ and suppose

$$
\sum_{j=1}^{m} \xi_{j} t^{j} \geq 0 \quad \text { for all } t \in \mathbb{R} .
$$

Let $\left(x_{1}, x_{2}, \ldots, x_{m}\right) \in \mathcal{W}_{m}$. Then there exists $u \in C^{\alpha}([0,2 \pi])$ such that

$$
x_{j}=\frac{1}{2 \pi} \int_{0}^{2 \pi}\left[u\left(e^{\imath \theta}\right)\right]^{j} d \theta
$$

for $1 \leq j \leq m$. But then

$$
\begin{aligned}
\sum_{j=1}^{m} \xi_{j} x_{j} & =\sum_{j=1}^{m} \xi_{j}\left[\frac{1}{2 \pi} \int_{0}^{2 \pi}\left[u\left(e^{\imath \theta}\right)\right]^{j} d \theta\right] \\
& =\frac{1}{2 \pi} \int_{0}^{2 \pi}\left(\sum_{j=1}^{m} \xi_{j}\left[u\left(e^{\imath \theta}\right)\right]^{j}\right) d \theta \\
& \geq 0 .
\end{aligned}
$$

This shows that

$$
\mathcal{W}_{m} \subset \Gamma_{m}
$$


To study the reverse inclusion, let $\left(x_{1}, x_{2}, \ldots, x_{m}\right)$ be a point in $\Gamma_{m}$. Then there are real numbers $t_{1}, \ldots, t_{n}$ and strictly positive numbers $\lambda_{1}, \ldots, \lambda_{n}$ such that

$$
\sum_{k=1}^{n} \lambda_{k}=1
$$

and

$$
\sum_{k=1}^{n} \lambda_{k} t_{k}^{j}=x_{j} \quad \text { for } 1 \leq j \leq m
$$

Divide the interval $[0,2 \pi]$ into $n$ subintervals $\left\{I_{k}\right\}$ with the length of $I_{k}$ equaling $2 \pi \lambda_{k}$. Set

$$
u_{0}(t)=t_{k} \quad \text { for } t \in I_{k} .
$$

Note that

$$
\int_{0}^{2 \pi}\left[u_{0}(t)\right]^{j} d t=\sum_{k=1}^{n} \lambda_{k} t_{k}^{j}=x_{j} .
$$

The function $u_{0}$ is not in the class $C^{\alpha}$ unless $n=1$. However since we can approximate $u_{0}$ in the $L^{m}$ norm by smooth functions, and since the Hilbert transform is continuous in $L^{m}$, the point $\left(x_{1}, \ldots, x_{m}\right)$ is in the closure of $\mathcal{W}_{m}$. This establishes Lemma 7.3 and completes the proof of the first two parts of Theorem 7.2.

To establish the third part of Theorem 7.2, we compute the differential of the mapping $F: C^{\alpha} \rightarrow \mathbb{R}^{m}$ given by

$$
F(u)=\left(\frac{1}{2 \pi} \int_{0}^{2 \pi} u(t) d t, \frac{1}{2 \pi} \int_{0}^{2 \pi}[u(t)]^{2} d t, \ldots, \frac{1}{2 \pi} \int_{0}^{2 \pi}[u(t)]^{m} d t\right) .
$$

The differential of $F$ is given by

$$
\begin{aligned}
& D F_{u}(h) \\
& =\left(\frac{1}{2 \pi} \int_{0}^{2 \pi} h(t) d t, \frac{1}{2 \pi} \int_{0}^{2 \pi} 2 u(t) h(t) d t, \ldots, \frac{1}{2 \pi} \int_{0}^{2 \pi} m u(t)^{m-1} h(t) d t\right) .
\end{aligned}
$$

If this is not a surjective map, then there exists $\left(\xi_{1}, \ldots, \xi_{m}\right) \in \mathbb{R}^{m}$ so that

$$
\int_{0}^{2 \pi}\left[\sum_{j=1}^{m} j \xi_{j} u(t)^{j-1}\right] h(t) d t=0
$$


for all $h$ of small $C^{\alpha}$ norm. This implies that

$$
\sum_{j=1}^{m} j \xi_{j} u(t)^{j-1} \equiv 0
$$

for all $\mathrm{t}$, and hence the function $u$ takes on only the finite number of values given by the roots of the polynomial $p(x)=\sum_{j=1}^{m} j \xi_{j} x^{j-1}$. Since $u$ is continuous, this can only happen if $u$ is the constant function given by the average value of the function $u$ over the interval $0 \leq t \leq 2 \pi$. In this case, the resulting analytic disc is a constant whose point image is contained in $T_{m}$. Therefore, $\operatorname{Disc}\left(T_{m}\right)-T_{m}$ is open, as desired.

\section{The Polynomial Hull of $R_{m}$.}

Recall that $R_{m}$ is defined as

$$
R_{m}=\left\{\left.\left(w, z_{1}, \ldots, z_{m}\right) \in \mathbb{C}^{m+1}\left|\Re\left(z_{j}\right)=\right| w\right|^{2 j}, \quad 1 \leq j \leq m\right\} .
$$

The main theorem of this section essentially states that the closure of the disc set of $R_{m}$ is convex in the normal variables, $z_{1}, \ldots, z_{m}$ and logarithmically convex in the tangential variable, $w$.

Theorem 8.1. Suppose $w_{1}, \ldots, w_{L} \in \mathbb{C}$ and $0 \leq \lambda_{1}, \ldots, \lambda_{L} \leq 1$ with $\sum_{j=1}^{L} \lambda_{j}=1$. Then the point

$$
\left(\prod_{j=1}^{L} w_{j}^{\lambda_{j}}, \sum_{j=1}^{L} \lambda_{j}\left|w_{j}\right|^{2}+\imath y_{1}, \ldots, \sum_{j=1}^{L} \lambda_{j}\left|w_{j}\right|^{2 m}+\imath y_{m}\right) \in \mathbb{C}^{m+1}
$$

belongs to the closure of the disc set of $R_{m}$.

Note that with $w_{1}=0$ and $\lambda_{1}=0$, the theorem implies that the slice of the disc set over the origin contains the slice of the convex hull of $R_{m}$ which lies over the origin.

Proof. We first consider the case when one of the $w_{j}$, say $w_{1}$, is zero. Define $\mathcal{M}: C^{\alpha}[0,1] \rightarrow \mathbb{R} \times \mathbb{R}^{m}$ by

$$
\mathcal{M}(u)=\left(0, \int_{0}^{1}(u(t))^{2} d t, \ldots, \int_{0}^{1}(u(t))^{2 m} d t\right) .
$$

Here $C^{\alpha}[0,1]$ denotes the space of real valued functions satisfying a Hölder condition of order $\alpha$. Since the defining equations for $R_{m}$ are independent of $\operatorname{Im} z_{j}=y_{j}, 1 \leq j \leq m$, it suffices to show that any point of the form

$$
q=\left(0, \sum_{j=1}^{L} \lambda_{j}\left|w_{j}\right|^{2}, \ldots, \sum_{j=1}^{L} \lambda_{j}\left|w_{j}\right|^{2 m}\right)
$$


can be approximated by $\mathcal{M}(g)$ where $g=|f|$ and $f$ is the boundary values (on the unit circle) of an analytic function that vanishes at the origin. To do this, we first repeat the arguments of Section 7 to show that $q$ can be approximated by $\mathcal{M}(u)$ for an appropriate $u \in C^{\alpha}[0,1]$. Next we approximate $u$ in the $L^{2 m}[0,1]$ norm by real-valued trigonometric polynomials of the form

$$
u_{N}(t)=\sum_{n=-N}^{N} a_{n} e^{2 \pi \imath n t} .
$$

By writing $u^{2 k}-u_{N}^{2 k}$ as $\left(u-u_{N}\right) P_{2 k-1}\left(u, u_{N}\right)$ where $P_{2 k-1}$ is a polynomial of degree $2 k-1$, and then using Hölder's inequality, we obtain

$$
\int_{0}^{1}(u(t))^{2 k}-\left(u_{N}(t)\right)^{2 k} d t \leq\left\|u-u_{N}\right\|_{L^{2 k}}\left\|P_{k}\left(u, u_{N}\right)\right\|_{L^{\frac{2 k}{2 k-1}}} .
$$

Since $k \leq m$ and $u_{N}$ is bounded in $L^{2 m}$ norm, we see that $\mathcal{M}\left(u_{N}\right)$ approximates $\mathcal{M}(u)$. Next, let

$$
F_{N}(\zeta)=\zeta^{N+1} \sum_{n=-N}^{N} a_{n} \zeta^{n} .
$$

Clearly, $F_{N}$ is holomorphic on the unit disc and $F_{N}(\zeta=0)=0$. Let $g_{N}(t)=\left|F_{N}\left(e^{2 \pi \imath t}\right)\right|$. Since $\mathcal{M}\left(g_{N}\right)=\mathcal{M}\left(u_{N}\right)$, the point $q$, given above, is approximated by $\mathcal{M}\left(g_{N}\right)$, and $g_{N}$ is the boundary values of the modulus of the analytic function, $F_{N}$, which vanishes at the origin. Therefore, $q$ is approximated by the center of the resulting analytic disc with boundary in $M$.

Now we consider the case where all the $w_{j}$ are nonzero. Let $t_{j}=\left|w_{j}\right|$. As in Section 7, divide the interval $0 \leq t \leq 1$ into disjoint subintervals $I_{j}$ of length $\lambda_{j}$. Define the step function $u$ to have value $t_{j}$ on the interval $I_{j}$. Then

$$
\int_{0}^{1} u(t)^{2 k} d t=\sum_{j=1}^{L} \lambda_{j} t_{j}^{2 k} .
$$

Let $a_{n}$ be the $n^{t h}$ Fourier coefficient of the function $\log (u)$ :

$$
a_{n}=\int_{0}^{1} \log (u(t)) e^{-2 \pi \imath n t} d t
$$

and consider the following polynomial

$$
f_{N}(\zeta)=a_{0}+\sum_{n=1}^{N} a_{n} \zeta^{n}
$$


Note that $\operatorname{Re} f_{N}\left(e^{2 \pi \imath t}\right)$ converges to $\ln (u(t))$ in the $L^{p}$ norm for any $p<\infty$.

Let $\alpha$ be an argument of $\prod_{j=1}^{L} w_{j}^{\lambda_{j}}$. Define

$$
F_{N}(\zeta)=\exp \left[\imath \alpha+f_{N}(\zeta)\right]
$$

Note that $\int_{0}^{1}(u(t))^{2 k} d t$ is approximated by

$$
\int_{0}^{1}\left|F_{N}\left(e^{2 \pi \imath t}\right)\right|^{2 k} d t=\int_{0}^{1} \exp \left(2 k \operatorname{Re} F_{N}\left(e^{2 \pi \imath t}\right)\right) d t .
$$

Also note that $F_{N}(\zeta=0)=e^{\imath \alpha+a_{0}}$ which by the formula for $a_{0}$ is

$$
\exp \left(\imath \alpha+\int_{0}^{1} \ln (u(t)) d t\right)=\exp \left(\imath \alpha+\sum_{j=1}^{L} \lambda_{j} \ln \left(t_{j}\right)\right)=\prod_{j=1}^{L} w_{j}^{\lambda_{j}} .
$$

Therefore, the point

$$
q=\left(\prod_{j=1}^{L} w_{j}^{\lambda_{j}}, \sum_{j=1}^{L} \lambda_{j}\left|w_{j}\right|^{2}, \ldots, \sum_{j=1}^{L} \lambda_{j}\left|w_{j}\right|^{2 m}\right)
$$

is approximated (as $N \rightarrow \infty$ ) by the center of the analytic disc that is generated by the analytic function $f_{N}$. This completes the proof of the theorem.

\section{Discs for the Free Model $F_{m}$.}

Recall that $F_{m}$ is defined as $F_{m}=\left\{(z, W) \in \mathbb{C} \times M_{m}^{\mathbb{C}} \mid \Re(W)=\Phi_{0}(z)\right\}$. Thus, analytic discs, $A=(f, g): D \rightarrow \mathbb{C} \times M_{m}^{\mathbb{C}}$ for $F_{m}$ must satisfy the equation

$$
\Re(g(\zeta))=\Phi_{0}(f(\zeta)) \text { for }|\zeta|=1 .
$$

Written out in components, this equation becomes

$$
\frac{g_{j k}(\zeta)+\overline{g_{k j}(\zeta)}}{2}=f(\zeta)^{j} \overline{f(\zeta)}^{k}
$$

This equation determines the boundary values of $\Re(g)$ on the unit circle. Via the Hilbert transform, this equation also determines the boundary values of $\Im(g)$ up to a constant matrix $Y \in H_{m}$. Note that the matrices $\operatorname{Re}(g)$ and $\operatorname{Im}(g)$ are related to $\Re(g)$ and $\Im(g)$ via the complex linear map $Q$ defined in Section 4.3. Therefore, the Hilbert transform used here is the traditional Hilbert transform factored through the map $Q$. 
As with $T_{m}$ and $R_{m}$, we are interested in the disc set of $F_{m}$. By the Poisson formula, the value of the analytic $\operatorname{disc} A$ at the origin is given by

$$
A(\zeta=0)=\left(f(0), \widetilde{\Phi_{0}}(f)+\imath Y\right)
$$

where the $j, k^{t h}$ entry of the Hermitian matrix $\widetilde{\Phi_{0}}(f)$ is the term

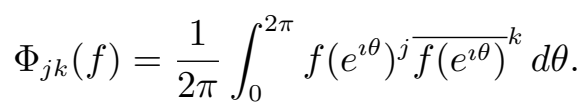

In this section, we examine the slice of the disc set of $F_{m}$ which lies in the normal space to $F_{m}$ at the origin. Using the group action for $F_{m}$, one can then obtain information about the disc set near other points of $F_{m}$. In order for the point $A(\zeta=0)$ to belong to the normal space to $F_{m}$ above the origin, we must have $f(0)=0$ and $Y=0$. Thus, the subset of the normal space to $F_{m}$ above the origin swept out by centers of analytic discs of class $C^{\alpha}$ with boundaries in $F_{m}$ is the set

$\mathcal{V}_{m}^{0}=\left\{X \in H_{m} \mid\right.$ there exists a holomorphic function $f$ with boundary values of class $C^{\alpha}$ and $f(0)=0$ such that $\left.X=\widetilde{\Phi_{0}}(f)\right\}$.

We must try to characterize the "moment" matricies $\left[\Phi_{j k}(f)\right], 1 \leq j, k \leq m$, where

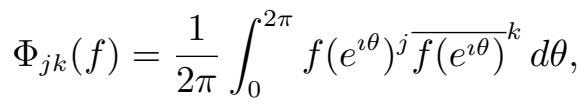

where $f$ is the boundary values of a holomorphic function in the unit disc $D$ with $f(0)=0$.

This extra condition on the measure restricts the range of the moment matrices. First, note from Schwarz' inequality that

$$
\sum_{j, k=1}^{m} \Phi_{j k}(f) \bar{\xi}_{j} \xi_{k}=\frac{1}{2 \pi} \int_{0}^{2 \pi}\left|\sum_{j=1}^{m} \bar{\xi}_{j} f\left(e^{\imath \theta}\right)^{j}\right|^{2} d \theta \leq|\xi|^{2} \sum_{j=1}^{m} \Phi_{j j}(f) .
$$

This inequality states that the norm of the moment matricies for the manifold $F_{m}$ is dominated "from above" by the norm of the moment matricies for the radial case. The interesting fact, displayed by the next theorem (when $p=2$ ), is that the above inequality can be reversed (up to a uniform constant factor). Therefore, the moment matrix for the free case is controlled, from above and below, by the moment matrix for the radial case. As we shall see, this theorem will imply that the range of the moment matrices for $F_{m}$ is smaller than the convex hull of $F_{m}$. 
Even though $p=2$ is the main case of interest we state and prove the following theorem for any $0<p<\infty$.

Theorem 9.1. Let $0<p<\infty$ and let $n$ be a positive integer. Then there is a positive constant $c(p, n)$ so that if $f \in H^{p n}$ and if $\left(\xi_{1}, \ldots, \xi_{n}\right) \in \mathbb{C}^{n}$, then

$$
\int_{0}^{2 \pi}\left|\sum_{j=1}^{n} \xi_{j} f\left(e^{\imath \theta}\right)^{j}\right|^{p} d \theta \geq \epsilon(p, n) \sum_{j=1}^{n}\left|\xi_{j}\right|^{p} \int_{0}^{2 \pi}\left|f\left(e^{\imath \theta}\right)\right|^{p j} d \theta .
$$

Here, the space $H^{q}$ refers to the space of analytic functions on the disc in the Hardy space with exponent $q$.

We introduce the following notation. Let $g$ be an entire function such that $g(0)=0$. For $\lambda>0$ set

$$
\Sigma(g, \lambda)=\{\zeta \in \mathbb{C}|| g(\zeta) \mid<\lambda\}
$$

and let $\Sigma_{0}(g, \lambda)$ be the connected component of $\Sigma(g, \lambda)$ containing the origin.

Lemma 9.2. There is a constant $C_{n}$ so that if $P(\zeta)=\sum_{j=1}^{n} a_{j} \zeta^{j}$ is a polynomial of degree $n$ vanishing at the origin, then for all $\lambda>0$,

$$
\Sigma_{0}(P, \lambda) \subset\left\{\zeta \in \mathbb{C}|| \zeta \mid<C_{n} \mu_{P}(\lambda)\right\}
$$

where $\mu_{P}(\lambda)>0$ satisfies

$$
\sum_{j=1}^{n}\left|a_{j}\right| \mu_{P}(\lambda)^{j}=\lambda
$$

Proof. Given the polynomial $P$ and $\lambda>0$, let $Q(w)=\frac{1}{\lambda} P\left(\mu_{P}(\lambda) w\right)$ so that $P(\zeta)=\lambda Q\left(\frac{\zeta}{\mu_{P}(\lambda)}\right)$. Then

$$
\Sigma_{0}(P, \lambda)=\mu_{P}(\lambda) \Sigma_{0}(Q, 1)
$$

Thus it suffices to show that if $Q(w)=\sum_{j=1}^{n} b_{j} w^{j}$ is a polynomial of degree $n$ vanishing at the origin, with $\sum_{j=1}^{n}\left|b_{j}\right|=1$, then $\Sigma_{0}(Q, 1)$ is contained in a fixed disc independent of $Q$.

Let $A=\sup |\zeta|$ where the supremum is taken over $\Sigma_{0}(Q, 1)$. Then we can find a curve in the complex plane going from the origin to a point $\zeta_{n}$ with $\left|\zeta_{n}\right|=A$, with the property that $|Q| \leq 1$ along this curve. Hence we can find points $\zeta_{1}, \zeta_{2}, \ldots, \zeta_{n}$ such that

(1) $\left|Q\left(\zeta_{j}\right)\right| \leq 1$ for all $j$; 
(2) $\left|\zeta_{j}\right|=\frac{j A}{n}$ for all $j$;

(3) $\left|\zeta_{j}-\zeta_{k}\right| \geq \frac{1}{n} A$ for $j \neq k$.

Now view the equations

$$
Q\left(\zeta_{k}\right)=\sum_{j=1}^{n} b_{j}\left(\zeta_{k}\right)^{j}, \quad 1 \leq k \leq n
$$

as a system of $n$ linear equations in the $n$ unknowns $\left\{b_{1}, \ldots, b_{n}\right\}$. Using Cramer's rule, we see that $b_{j}=D_{j} / D_{0}$ where

$$
D_{0}=\left|\begin{array}{cccccc}
\zeta_{1} & \zeta_{1}^{2} & \cdots & \zeta_{1}^{k} & \cdots & \zeta_{1}^{n} \\
\zeta_{2} & \zeta_{2}^{2} & \cdots & \zeta_{2}^{k} & \cdots & \zeta_{1}^{n} \\
\vdots & \vdots & \ddots & \vdots & \ddots & \vdots \\
\zeta_{n} & \zeta_{n}^{2} & \cdots & \zeta_{n}^{k} & \cdots & \zeta_{n}^{n}
\end{array}\right|
$$

and $D_{j}$ is obtained from $D_{0}$ by replacing the $\zeta_{k}^{j}$ in the $j^{\text {th }}$ column by $Q\left(\zeta_{k}\right)$. We obtain a bound

$$
\left|D_{j}\right| \leq C_{n} A^{\frac{n(n+1)}{2}-j}
$$

while the Vandermonde determinant shows that

$$
\left|D_{0}\right| \geq c_{n} A^{\frac{n(n+1)}{2}}
$$

Since $\sum_{j=1}^{n}\left|b_{j}\right|=1$, this gives us a bound from above on $A$ and proves Lemma 9.2

We now turn back to the proof of Theorem 9.1. For a function $F$ holomorphic in the unit disc $D$, we let

$$
N F\left(e^{\imath \theta}\right)=\sup _{\Gamma_{\theta}}|F(z)|
$$

where $\Gamma_{\theta}$ is a nontangential approach region to the boundary point $e^{\imath \theta}$. Recall the theorem of Burkholder, Gundy, and Silverstein which says that for $F \in H^{p}(D)$,

$$
\int_{0}^{2 \pi}\left|F\left(e^{\imath \theta}\right)\right|^{p} d \theta \approx \int_{0}^{2 \pi}\left|N F\left(e^{\imath \theta}\right)\right|^{p} d \theta
$$

for $0<p<\infty$. 
Let $f$ be holomorphic in a neighborhood of the closed unit disc, with $f(0)=0$, and let $\left\{\xi_{1}, \ldots, \xi_{n}\right\} \in \mathbb{C}^{n}$. Put $F(z)=\sum_{j=1}^{n} \xi_{j} f(z)^{j}$. Then

$$
\begin{aligned}
\int_{0}^{2 \pi}\left|\sum_{j=1}^{n} \xi_{j} f\left(e^{\imath \theta}\right)^{j}\right|^{p} d \theta & =\int_{0}^{2 \pi}\left|F\left(e^{\imath \theta}\right)\right|^{p} d \theta \\
& \geq c_{p} \int_{0}^{2 \pi}\left|N F\left(e^{\imath \theta}\right)\right|^{p} d \theta \\
& =c_{p} p \int_{0}^{\infty} \lambda^{p-1} m\left\{\theta|| N F\left(e^{\imath \theta}\right) \mid>\lambda\right\} d \lambda .
\end{aligned}
$$

Put

$$
E_{\lambda}=\left\{e^{\imath \theta}|| N F\left(e^{\imath \theta}\right) \mid>\lambda\right\}
$$

Then $E_{\lambda}$ is a countable union of open intervals on the unit circle. Let $T_{\lambda}$ be the union of the "tents" over these intervals, and let $W_{\lambda}=D \backslash T_{\lambda}$. Then $|F(z)| \leq \lambda \quad z \in W_{\lambda}$. Put $P(\zeta)=\sum_{j=1}^{n} \xi_{j} \zeta^{j}$, so that $F(z)=P(f(z))$. Thus for $z \in W_{\lambda},|P(f(z))| \leq \lambda$, and since $W_{\lambda}$ is connected and $f(0)=0$, it follows that $f(z) \in \Sigma_{0}(P, \lambda)$. By the lemma, it follows that

$$
|f(z)| \leq C_{n} \mu_{P}(\lambda) \quad \text { for } z \in W_{\lambda} .
$$

Hence

$$
\left\{e^{\imath \theta}|| N F\left(e^{\imath \theta}\right) \mid>\lambda\right\} \supset\left\{e^{\imath \theta}|| N f\left(e^{\imath \theta}\right) \mid>C_{n} \mu_{P}(\lambda)\right\} .
$$

It follows that

$$
\int_{0}^{2 \pi}\left|\sum_{j=1}^{n} \xi_{j} f\left(e^{\imath \theta}\right)^{j}\right|^{p} d \theta \geq p c_{p} \int_{0}^{\infty} \lambda^{p-1} m\left\{e^{\imath \theta}|| N f\left(e^{\imath \theta}\right) \mid>C_{n} \mu_{P}(\lambda)\right\} d \lambda .
$$

In this last integral, make the change of variables $\lambda=\sum_{j=1}^{n}\left|\xi_{j}\right|\left(\frac{s}{C_{n}}\right)^{j}$ so that $\mu_{P}(\lambda)=\frac{s}{C_{n}}$. It follows that 
$(9.21)$

$$
\begin{aligned}
\int_{0}^{2 \pi}\left|\sum_{j=1}^{n} \xi_{j} f\left(e^{\imath \theta}\right)^{j}\right|^{p} d \theta & \geq c(p, n) \int_{0}^{\infty}\left[\sum_{j=1}^{n}\left|\xi_{j}\right| s^{j}\right]^{p} m\left\{\theta \mid N f\left(e^{\imath \theta}\right)>s\right\} \frac{d s}{s} \\
& \geq c(p, n) \sum_{j=1}^{n}\left|\xi_{j}\right|^{p} \int_{0}^{\infty} s^{p j-1} m\left\{\theta \mid N f\left(e^{\imath \theta}\right)>s\right\} d s \\
& =c(p, n) \sum_{j=1}^{n}\left|\xi_{j}\right|^{p} \int_{0}^{2 \pi}\left|N f\left(e^{\imath \theta}\right)\right|^{p j} d \theta \\
& \approx c(p, n) \sum_{j=1}^{n}\left|\xi_{j}\right|^{p} \int_{0}^{2 \pi}\left|f\left(e^{\imath \theta}\right)\right|^{p j} d \theta .
\end{aligned}
$$

This completes the proof of the theorem.

We conclude with two examples. The first example shows that the conclusions of Theorem 9.1 are not true if $f$ is not the boundary values of a holomorphic function. The second example shows that Theorem 9.1 implies that the disc set of $F_{m}$ is strictly smaller than its convex hull.

Example 9.3. Set

$$
f_{n}(x)= \begin{cases}\alpha, & \text { for } 0 \leq x \leq \frac{1}{n} \\ -\frac{\alpha}{n-1}, & \text { for } \frac{1}{n}<x \leq 1\end{cases}
$$

Then

$$
\int_{0}^{1} f_{n}(x) d x=0
$$

Put $\xi_{1}=\alpha$, and $\xi_{2}=-1$. Then an easy computation shows that

$$
\int_{0}^{1}\left|\xi_{1} f(x)+\xi_{2} f(x)^{2}\right|^{2} d x \approx \frac{\alpha^{4}}{n^{2}}
$$

while

$$
\left|\xi_{1}\right|^{2} \int_{0}^{1}|f(x)|^{2} d x+\left|\xi_{2}\right|^{2} \int_{0}^{1}|f(x)|^{4} d x \approx \frac{\alpha^{4}}{n} .
$$

As $n$ gets large, it is clear that the conclusions of Theorem 9.1 are not true for this function $f$ (which, of course, is not holomorphic).

Example 9.4. We show that the disc set of $U_{2}$ is strictly smaller than the convex hull of $U_{2}$. We define $\Phi: \mathbb{C} \rightarrow U_{2}$ by

$$
\Phi(z)=\left(\begin{array}{ll}
|z|^{2} & z \bar{z}^{2} \\
z^{2} \bar{z}|z|^{4}
\end{array}\right) .
$$


We let $M^{\epsilon}$ be the convex combination $\lambda_{\epsilon} \Phi(\epsilon)+\left(1-\lambda_{\epsilon}\right) \Phi\left(\frac{-1}{\epsilon}\right)$, where $\lambda_{\epsilon}=\frac{1}{\epsilon^{2}+1}$ (so $\left(0, M^{\epsilon}\right)$ belongs to the convex hull of $\left.U_{2}\right)$. Let $m_{j k}^{\epsilon}$ denote the $j, k^{\text {th }}$ entry of $M^{\epsilon}$. After a computation, we have

$$
\begin{aligned}
& \sum_{j, k=1}^{2} m_{j k}^{\epsilon} \bar{\xi}_{j} \xi_{k} \\
& =\left[\left(1+\epsilon^{2}\right)\left|\xi_{1}\right|^{2}+2 \operatorname{Re}\left\{\left(\epsilon^{3}-\frac{1}{\epsilon}\right) \xi_{1} \bar{\xi}_{2}\right\}+\left(\epsilon^{4}+\frac{1}{\epsilon^{2}}\right)\left|\xi_{2}\right|^{2}\right]\left(1+\epsilon^{2}\right)^{-1}
\end{aligned}
$$

and

$$
\sum_{j=1}^{2} m_{j j}^{\epsilon}\left|\xi_{j}\right|^{2}=\left[\left(1+\epsilon^{2}\right)\left|\xi_{1}\right|^{2}+\left(\epsilon^{4}+\frac{1}{\epsilon^{2}}\right)\left|\xi_{2}\right|^{2}\right]\left(1+\epsilon^{2}\right)^{-1}
$$

If $m_{j k}^{\epsilon}=\Phi_{j k}(f)$ for some analytic function $f$ with $C^{\alpha}$ boundary values, then Theorem 9.1 implies

$$
\sum_{j, k=1}^{2} m_{j k}^{\epsilon} \bar{\xi}_{j} \xi_{k} \geq c \sum_{j=1}^{2} m_{j j}^{\epsilon}\left|\xi_{j}\right|^{2}
$$

where $c$ is a uniform positive constant. Set $\xi_{1}=\frac{1}{\epsilon}$ and $\xi_{2}=1$. Then $\sum_{j, k=1}^{2} m_{j k}^{\epsilon} \bar{\xi}_{j} \xi_{k}$ stays bounded in $\epsilon$ for $0<\epsilon<1$, whereas $\sum_{j=1}^{2} m_{j j}^{\epsilon}\left|\xi_{j}\right|^{2} \approx \frac{1}{\epsilon^{2}}$. This contradicts the above inequality when $\epsilon$ is small. Thus, for $\epsilon$ sufficiently small, $m_{j k}^{\epsilon}$ cannot be written as $\Phi_{j k}(f)$ for an analytic function $f$ with $C^{\alpha}$ boundary values.

\section{References}

[BRT] M.S. Baouendi, L.P. Rothschild and F. Treves, CR structures with group action and extendability of CR functions, Invent. Math., 82 (1985), 359-396.

[B] A. Boggess, CR Manifolds and the tangential Cauchy-Riemann complex, CRC Press, Bocca Raton, Ann Arbor, Boston, 1991.

[BN1] A. Boggess and A. Nagel, Local reproducing kernels on wedge-like domains with type 2 edges, Pacific J. of Math., 167 (1995), 1-47.

[BN2] - Representing measures for holomorphic functions on type 2 wedges in 'Essays on Fourier Analysis in Honor of Elias M. Stein' (C. Fefferman, R. Fefferman and S. Wainger, eds), Princeton University Press, Princeton, New Jersey, (1995), 69-82.

[BD] A. Boivin and R. Dwilewicz, Extension and approximation of CR functions on tube manifolds, preprint.

[FS] G.B. Folland and E.M. Stein, Estimates for the $\bar{\partial}_{b}$-complex and analysis on the Heisenberg group, Comm. Pure Appl. Math., 27 (1974), 429-522. 
[H] L. Hörmander, An Introduction to Complex Analysis in Several Variables, North Holland Publishing, Amsterdam, London, 1973.

Received May 8, 1996. The last author was supported in part by a grant from the National Science Foundation.

TEXAS A\&M UNIVERSITY

College Station, TX 77843-3368

E-mail address: boggess@math.tamu.edu

PRINCETON UNIVERSITY

AND

UNIVERSITY OF WISCONSIN-MADISON

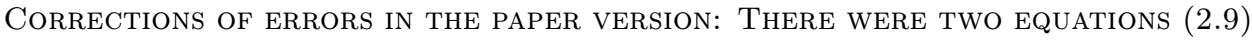
IN THE PAPER VERSION. BOTH EQUATION NUMBERS HAVE BEEN REMOVED. There WERE Also two Equations (6.7). The SECOND has BeEn Changed to (6.11). 